\title{
Expectativa de vida com incapacidade funcional em idosos em São Paulo, Brasil ${ }^{1}$
}

\author{
Mirela Castro Santos Camargos, ${ }^{2}$ Ignez Helena Oliva Perpétuo ${ }^{2}$ \\ e Carla Jorge Machado ${ }^{2}$
}

Como citar Camargos MCS, Perpétuo IHO, Machado CJ. Expectativa de vida com incapacidade funcional em idosos em São Paulo, Brasil. Rev Panam Salud Publica. 2005;17(5/6):379-86.

RESUMO Objetivo. Determinar, para indivíduos com 60 anos ou mais no ano de 2000, no Município de São Paulo, por sexo e idade, a expectativa de vida livre de e com incapacidade funcional e, neste último caso, mensurar os anos a serem vividos com e sem dependência.

Método. As estimativas de expectativa de vida livre de incapacidade funcional, expectativa de vida com incapacidade funcional, expectativa de vida com incapacidade funcional e sem dependência e expectativa de vida com incapacidade funcional e dependência foram geradas a partir da construção de uma tabela de sobrevivência, conforme o método descrito por Sullivan. Os dados básicos utilizados para o cálculo das expectativas foram o número estimado de idosos no Município em meados de 2000, obtido a partir dos censos demográficos de 1991 e 2000, e as informações sobre óbitos na população idosa, obtidas da Fundação Sistema Estadual de Análise de Dados (SEADE). As taxas de prevalência de incapacidade funcional e dependência foram calculadas a partir dos dados sobre as atividades de vida diária do Projeto Saúde, BemEstar e Envelhecimento na América Latina e Caribe ( $S A B E$ ). As atividades de vida diária contempladas no $S A B E$ são: vestir-se, comer, tomar banho, ir ao banheiro, deitar-se e levantar da cama e atravessar um cômodo da casa. A incapacidade funcional foi definida como a dificuldade em realizar uma ou mais atividades de vida diária. A dependência foi definida como a necessidade de auxilio para realizar pelo menos uma atividade.

Resultados. Em 2000, ao atingir os 60 anos, os homens paulistanos podiam esperar viver, em média, 17,6 anos, dos quais 14,6 (83\%) seriam vividos livres de incapacidade funcional. As mulheres na mesma idade podiam esperar viver 22,2 anos, dos quais 16,4 anos (74\%) seriam livres de incapacidade funcional. Dos anos com incapacidade funcional, os homens viveriam 1,6 ano (9\%) com dependência, contra 2,5 anos (11\%) para as mulheres.

Conclusão. Apesar de as mulheres idosas paulistanas terem apresentado maior expectativa de vida do que os homens, foi menor a proporção de anos vividos livres de incapacidade funcional. O número de anos com incapacidade funcional e dependência também foi maior entre as mulheres. As politicas públicas devem levar em conta as diferentes necessidades das mulheres e homens idosos, assim como outras especificidades dessa população.

Palavras-chave Envelhecimento da população, qualidade de vida, saúde do idoso.

1 Trabalho baseado na dissertação "Estimativas de expectativa de vida livre de e com incapacidade funcional: uma aplicação do método de Sullivan para idosos paulistanos, 2000", apresentada ao Programa de Mestrado em Demografia do Centro de Desenvolvimento e Planejamento Regional (CEDEPLAR) da Universidade Federal de Minas Gerais (UFMG).
2 UFMG, CEDEPLAR, Departamento de Demografia. Enviar correspondência para Mirela Castro Santos Camargos no seguinte endereço: Rua São Jorge 125/401, Bairro Carlos Prates, CEP 30710110, Belo Horizonte, MG, Brasil. Telefone: +55-313272-8008; fax: +55-31-3201-3657; e-mail: mirela@ cedeplar.ufmg.br
A quantidade de anos vividos com e sem saúde e os tipos de problemas experimentados pelos idosos exercem um papel fundamental no uso dos serviços de saúde (1). Atualmente, quando se pensa em indivíduos ido- 
sos, as principais preocupações dizem respeito a quem cuidará dessa população crescente e a como serão vividos os anos ganhos com o aumento da expectativa de vida. Essa reflexão exige informações sobre os indicadores de saúde dessa população.

A noção de expectativa de vida saudável, proposta na década de 1960 (2) e desenvolvida nos anos 1970 (3), refere-se ao número médio de anos de vida que as pessoas de determinada idade podem esperar viver com saúde, desde que se mantenham as taxas de morbidade e mortalidade (4). A expectativa de vida saudável pode ser mensurada pelos métodos de Sullivan, da tábua de vida de duplo decremento e da tábua de vida multiestado $(5,6)$. Esses dois últimos métodos exigem dados longitudinais de incidência, raramente disponíveis, especialmente no caso de países em desenvolvimento. Assim, o método de Sullivan é o mais utilizado, pois usa dados correntes de prevalência de determinado estado de saúde (1, 5-8).

A expectativa de vida saudável calculada pelo método de Sullivan reflete a saúde corrente de uma população real ajustada pelos níveis de mortalidade e independe da estrutura etária da população (3). Para calculá-la, é necessário conhecer as proporções de pessoas "saudáveis" e "não-saudáveis" (que podem ser obtidas por meio de estudos transversais) em cada idade ou em determinado grupo etário, assim como a mortalidade em determinado período. Como os dados são derivados de uma tábua de vida de período, podem existir erros associados às estimativas. Entretanto, a experiência mostra que o método de Sullivan é vantajoso por sua simplicidade, precisão relativa e facilidade de interpretação (7). Além disso, desde que não ocorram grandes mudanças nas taxas de prevalência e de mortalidade ao longo do tempo, as estimativas encontradas são bastante semelhantes às obtidas por meio de dados longitudinais (8).

A expectativa de vida saudável pode ser definida de várias formas. A noção de vida saudável pode levar em conta, por exemplo, o bem-estar físico, mental ou social; as conseqüências de doenças; a incapacidade funcional; e o envelhecimento bem-sucedido (5). Na prática, a expectativa de vida saudável é comumente estimada por meio da mensuração da expectativa de vida livre de incapacidade funcional (9), ou seja, livre de dificuldade em executar atividades da vida diária.

A expectativa de vida livre de incapacidade funcional fornece informações não apenas sobre a prevalência de incapacidade funcional, mas também sobre a duração potencial da incapacidade e o tempo pelo qual a população vai precisar de cuidados pessoais (10). Dessa forma, contribui para o levantamento das necessidades da população idosa e fornece dados valiosos para a alocação adequada de recursos humanos e financeiros e para o planejamento de políticas públicas cujo foco seja o aumento do número de anos vividos com saúde pela população idosa.

Assim, o objetivo do presente estudo foi determinar, para indivíduos com 60 anos ou mais no ano de 2000, no Município de São Paulo, por sexo e idade, a expectativa de vida livre de e com incapacidade funcional e, neste último caso, mensurar os anos a serem vividos com e sem dependência.

\section{MATERIAIS E MÉTODOS}

As estimativas de expectativa de vida livre de incapacidade funcional, expectativa de vida com incapacidade funcional, expectativa de vida com incapacidade funcional e sem dependência e expectativa de vida com incapacidade funcional e dependência para os homens e as mulheres idosas (60 anos ou mais) do Município de São Paulo foram geradas a partir da construção de uma tabela de sobrevivência (tábua de vida), conforme o método descrito por Sullivan (3). O número de anos vividos dentro de cada grupo de idade na tábua de vida é distribuído segundo as prevalências de incapacidade funcional e dependência em cada grupo etário.

Partindo do pressuposto de que as taxas específicas de mortalidade $\left({ }_{n} M_{x}\right)$ no período se aplicam à coorte, foram empregadas as ${ }_{n} M_{x}$ correntes na população do Município de São Paulo em 2000, calculadas a partir de dados sobre a população no meio do período e dos óbitos ocorridos naquele ano. A população idosa estimada para o meio do ano de 2000 foi obtida a partir dos censos demográficos de 1991 (11) e 2000 (12), e as informações sobre óbitos na população idosa provieram da Fundação Sistema Estadual de Análise de Dados (SEADE) (13). Foram consideradas as médias dos óbitos registrados no triênio 1999/2000/2001, por sexo e faixa etária.

As taxas de prevalência de incapacidade funcional e dependência foram estimadas a partir dos dados sobre as atividades de vida diária do Projeto Saúde, Bem-Estar e Envelhecimento na América Latina e Caribe (SABE) (14), que coletou informações de idosos de sete países da América Latina e do Caribe: Argentina, Barbados, Brasil, Chile, Cuba, México e Uruguai. O Brasil foi representado pelo Município de São Paulo, onde foram realizadas entrevistas com 2143 idosos entre janeiro de 2000 e março de 2001, por meio de pesquisa domiciliar, não incluindo idosos institucionalizados (15).

As atividades de vida diária contempladas no questionário do SABE e empregadas na presente análise são: vestir-se, comer, tomar banho, ir ao banheiro, deitar-se e levantar da cama e atravessar um cômodo da casa. $\mathrm{O}$ idoso deveria responder se apresentava alguma dificuldade para realizar cada uma dessas atividades, com as seguintes opções de resposta: sim, não, não sabe ou não respondeu. Nos casos em que a resposta foi sim, investigouse se o idoso era ajudado por alguém para desempenhar aquela atividade, sendo as opções de resposta iguais às citadas anteriormente. Durante a entrevista, o idoso foi orientado a desconsiderar problemas com duração inferior a 3 meses, ou seja, foram consideradas apenas as situações crônicas.

A incapacidade funcional foi definida como a dificuldade em realizar uma ou mais atividades de vida diária. A dependência foi definida como a necessidade de auxílio para realizar pelo menos uma atividade. Os indivíduos 
foram classificados em: livres de incapacidade funcional, com incapacidade funcional, com incapacidade funcional e sem dependência e com incapacidade funcional e dependência. As informações foram processadas no programa Statistical Package for the Social Sciences (SPSS), versão 10.0.

Foram utilizados os pesos existentes na própria base de dados do SABE, para que os dados refletissem a realidade da população do Município de São Paulo em termos quantitativos. Colocou-se como critério de exclusão da amostra a presença da resposta "não sabe" ou "não respondeu" para todas as atividades de vida diária avaliadas. Apenas um sujeito foi excluído, ou seja, a amostra considerada neste estudo foi de 2142 idosos. Os três indivíduos que apresentaram esse tipo de resposta em menos de seis itens, mas que relataram ter dificuldade ou dependência em pelo menos uma atividade, foram mantidos na amostra, sendo classificados nos grupos com incapacidade funcional ou dependência.

\section{RESULTADOS}

As estimativas de expectativa de vida indicaram que, em 2000, as mulheres idosas paulistanas podiam esperar viver mais do que os homens (tabela 1). A diferença entre os sexos em relação ao número de anos a serem vividos (cerca de 5 anos aos 60 anos) diminuiu à medida que avançou a idade, chegando a cerca de 1 ano no último grupo analisado.

As figuras 1 e 2 permitem visualizar, dentro do total de anos a serem vividos a partir de cada idade exata, a proporção de anos livres de incapacidade funcional, com incapacidade funcional e sem dependência e com incapacidade funcional e dependência para homens e mulheres, respectivamente. Pode-se verificar que a distribuição proporcional é diferente entre os sexos. Entretanto, ambos apresentam uma característica comum: à medida que aumenta a idade, a porcentagem de anos a serem vividos livres de incapacidade funcional diminui, ao passo que os anos com incapacidade funcio-

TABELA 1. Expectativa de vida estimada para idades exatas a partir dos 60 anos segundo incapacidade funcional e dependência, São Paulo (SP), Brasil, 2000

\begin{tabular}{|c|c|c|c|c|c|}
\hline \multirow{2}{*}{$\begin{array}{l}\text { Sexo } \\
\text { e idade } \\
\text { (anos) }\end{array}$} & \multirow{2}{*}{$\begin{array}{l}\text { Expectativa } \\
\text { de vida total } \\
\text { (anos) }\end{array}$} & \multirow{2}{*}{$\begin{array}{l}\text { Expectativa de vida } \\
\text { livre de incapacidade } \\
\text { funcional (anos) }\end{array}$} & \multicolumn{3}{|c|}{$\begin{array}{c}\text { Expectativa de vida com } \\
\text { incapacidade funcional (anos) }\end{array}$} \\
\hline & & & Total & $\begin{array}{c}\text { Sem } \\
\text { dependência }\end{array}$ & $\begin{array}{c}\text { Com } \\
\text { dependência }\end{array}$ \\
\hline \multicolumn{6}{|l|}{ Homens } \\
\hline 60 & 17,6 & 14,6 & 3,0 & 1,4 & 1,6 \\
\hline 65 & 14,5 & 11,7 & 2,8 & 1,3 & 1,6 \\
\hline 70 & 11,7 & 9,0 & 2,7 & 1,3 & 1,5 \\
\hline 75 & 9,3 & 6,7 & 2,6 & 1,2 & 1,3 \\
\hline 80 & 7,2 & 4,9 & 2,3 & 0,9 & 1,4 \\
\hline 85 & 5,8 & 3,5 & 2,3 & 0,8 & 1,5 \\
\hline \multicolumn{6}{|l|}{ Mulheres } \\
\hline 60 & 22,2 & 16,4 & 5,8 & 3,4 & 2,5 \\
\hline 65 & 18,4 & 13,2 & 5,2 & 2,8 & 2,4 \\
\hline 70 & 14,9 & 10,0 & 4,9 & 2,4 & 2,5 \\
\hline 75 & 11,6 & 7,2 & 4,5 & 2,0 & 2,4 \\
\hline 80 & 8,9 & 4,8 & 4,0 & 1,5 & 2,5 \\
\hline 85 & 6,8 & 2,9 & 3,9 & 1,3 & 2,7 \\
\hline
\end{tabular}

Fonte dos dados básicos para o cálculo das expectativas: Instituto Brasileiro de Geografia e Estatística (IBGE) (11, 12), Fundação Sistema Estadual de Análise de Dados (SEADE) (13) e Projeto Saúde, Bem-Estar e Envelhecimento na América Latina e Caribe (SABE) (14).

a Incapacidade funcional: dificuldade de realizar uma ou mais atividades de vida diária (vestir-se, comer, tomar banho, ir ao banheiro, deitar-se e levantar da cama e atravessar um cômodo da casa). Dependência: necessidade de auxílio para realizar pelo menos uma atividade.

FIGURA 1. Proporção de anos a serem vividos livres de incapacidade funcional, com incapacidade funcional e sem dependência e com incapacidade funcional e dependência em relação à expectativa de vida total para homens de 60 anos ou mais, Município de São Paulo (SP), Brasil, 2000a

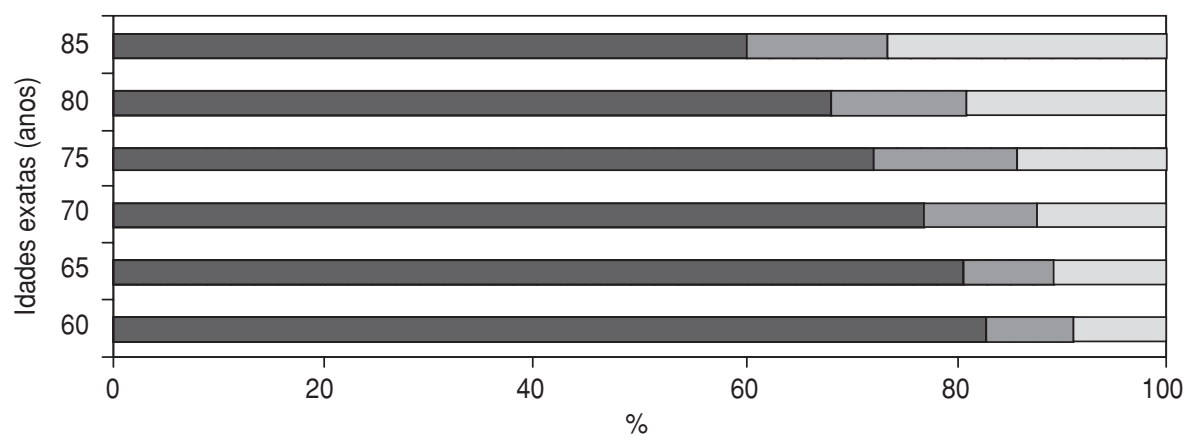

Expectativa de vida livre de incapacidade funcional

Expectativa de vida com incapacidade funcional e sem dependência

Expectativa de vida com incapacidade funcional e dependência

Fonte dos dados básicos para o cálculo das expectativas: Instituto Brasileiro de Geografia e Estatística (IBGE) $(11,12)$ Fundação Sistema Estadual de Análise de Dados (SEADE) (13) e Projeto Saúde, Bem-Estar e Envelhecimento na América Latina e Caribe (SABE) (14).

a Incapacidade funcional: dificuldade de realizar uma ou mais atividades de vida diária (vestir-se, comer, tomar banho, ir ao banheiro, deitar-se e levantar da cama e atravessar um cômodo da casa). Dependência: necessidade de auxílio para realizar pelo menos uma atividade.

nal e dependência aumentam. Esse comportamento é esperado, uma vez que as estimativas apresentadas se baseiam nas taxas de prevalência de in- capacidade funcional e dependência, que crescem com o avançar da idade.

A figura 3, por sua vez, permite visualizar a redução na proporção de 
anos a serem vividos livres de incapacidade funcional à medida que a idade aumenta. Quanto aos anos com incapacidade funcional, observa-se um comportamento inverso, ou seja, um crescimento com o avançar da idade. Para ambos os sexos, a proporção de anos a serem vividos com incapaci-
FIGURA 2. Proporção de anos a serem vividos livres de incapacidade funcional, com incapacidade funcional e sem dependência e com incapacidade funcional e dependência em relação à expectativa de vida total para mulheres com 60 anos ou mais, Município de São Paulo (SP), Brasil, 2000a

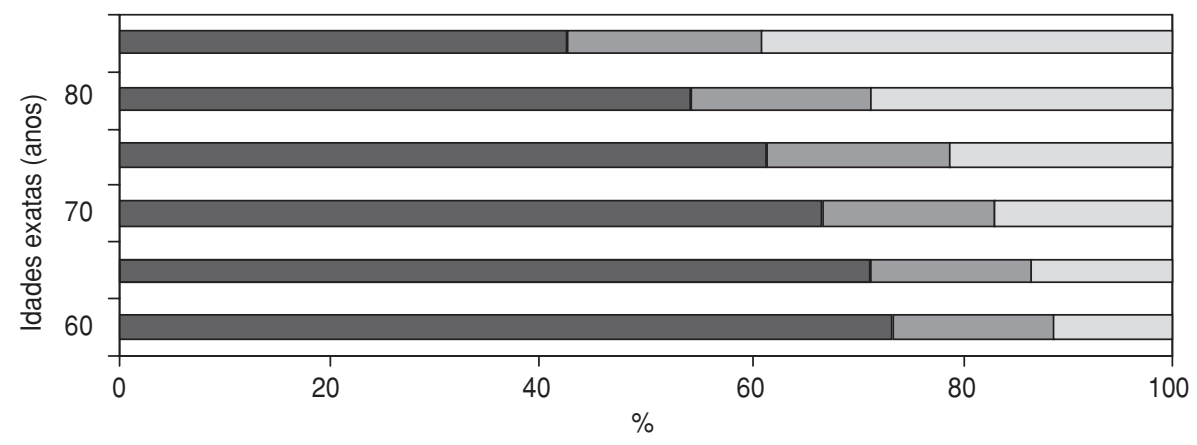

$\square$ Expectativa de vida livre de incapacidade funcional

$\square$ Expectativa de vida com incapacidade funcional e sem dependência

$\square$ Expectativa de vida com incapacidade funcional e dependência

Fonte dos dados básicos para o cálculo das expectativas: Instituto Brasileiro de Geografia e Estatística (IBGE) (11, 12), Fundação Sistema Estadual de Análise de Dados (SEADE) (13) e Projeto Saúde, Bem-Estar e Envelhecimento na América Latina e Caribe (SABE) (14)

a Incapacidade funcional: dificuldade de realizar uma ou mais atividades de vida diária (vestir-se, comer, tomar banho, ir ao banheiro, deitar-se e levantar da cama e atravessar um cômodo da casa). Dependência: necessidade de auxílio para realizar pelo menos uma atividade.

FIGURA 3. Proporção de expectativa de vida livre de incapacidade funcional e de expectativa de vida com incapacidade funcional em relação à expectativa de vida total por sexo e idades exatas, Município de São Paulo (SP), Brasil, 2000ª

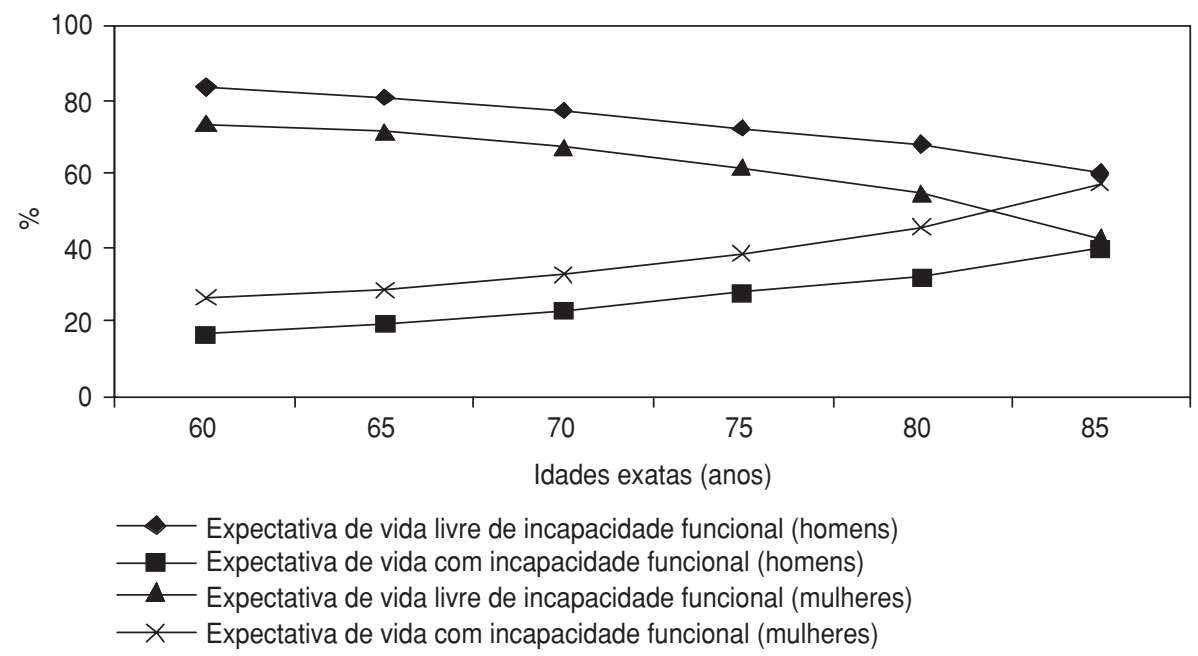

Fonte dos dados básicos para o cálculo das expectativas: Instituto Brasileiro de Geografia e Estatística (IBGE) (11, 12), Fundação Sistema Estadual de Análise de Dados (SEADE) (13) e Projeto Saúde, Bem-Estar e Envelhecimento na América Latina e Caribe (SABE) (14).

a Incapacidade funcional: dificuldade de realizar uma ou mais atividades de vida diária (vestir-se, comer, tomar banho, ir ao banheiro, deitar-se e levantar da cama e atravessar um cômodo da casa). dade funcional é sempre menor do que a proporção de anos a serem vividos sem essa condição, exceto para as mulheres de 85 anos.

No que se refere a diferenças entre os sexos, pode-se observar que, em todas as idades analisadas, os homens apresentam uma maior proporção de anos livres de incapacidade funcional se comparados às mulheres. Nota-se que as diferenças proporcionais entre os sexos em relação aos anos a serem vividos sem incapacidade aumentam quanto mais avançada a idade. Aos 85 anos, por exemplo, as mulheres podem esperar viver mais da metade dos anos que lhes restam com incapacidade funcional, ao passo que, para os homens, essa condição atinge apenas cerca de $40 \%$ dos anos a serem vividos. Assim, embora as mulheres tenham vantagem em quantidade de anos a serem vividos, a qualidade dos anos remanescentes fica comprometida pela dificuldade em desempenhar as atividades de vida diária.

Quanto aos anos a serem vividos com incapacidade funcional, observase que nem sempre os idosos vão necessitar de ajuda. Em relação ao total de anos a serem vividos, assim como ocorre com a expectativa de vida com incapacidade funcional para ambos os sexos, proporcionalmente, a expectativa de vida com incapacidade funcional e sem dependência tende a aumentar com a idade, com pequenas oscilações (figura 4). Entretanto, quando são considerados apenas os anos com incapacidade funcional (figura 5), em termos relativos, a expectativa de vida com incapacidade funcional e sem dependência tende a diminuir, comportamento mais evidente entre as mulheres. Assim, existe, por um lado, uma tendência de aumento na proporção de anos vividos com incapacidade funcional e, por outro, dentro desses anos, uma tendência à redução da independência.

Entre os homens idosos, o número de anos a serem vividos com incapacidade funcional e dependência é menor em relação às mulheres. Para ambos os sexos, os anos de vida remanescentes vão diminuindo com o aumento da idade, enquanto que a expectativa de 
vida com incapacidade funcional e dependência praticamente se mantém, com valores próximos em todas as idades analisadas (tabela 1). Assim, do total de anos remanescentes em cada idade, em cerca de 1,5 e 2,5 anos os homens e as mulheres, respectivamente, terão dificuldade para realizar as atividades de vida diária e dependerão da ajuda de outras pessoas. Em termos absolutos, os valores da expectativa de vida com incapacidade funcional para as diversas idades exatas e para ambos os sexos também não sofrem grandes variações, diferentemente da expectativa de vida livre de incapacidade funcional. Esse tipo de comportamento leva a crer que a expectativa de vida com incapacidade funcional e a expectativa de vida com incapacidade funcional e dependência não sofrem grandes variações com o avançar da idade.

$\mathrm{Se}$, em termos absolutos, as variações são pequenas, em termos relativos a proporção atribuída aos anos gastos com incapacidade funcional e dependência vai, logicamente, aumentando com o avançar da idade, sendo maior entre as mulheres em todas as idades analisadas (figura 4). A diferença entre os sexos, que se manteve em torno de 1 ano, também tende a crescer com o aumento da idade quando analisada em valores proporcionais, chegando a $12 \%$ aos 85 anos e mais, contra apenas $2 \%$ aos 60 anos.

Considerando apenas os anos a serem vividos com incapacidade funcional (figura 5), com exceção das duas primeiras idades analisadas para as mulheres, esses serão vividos, em sua maior parte, na dependência da ajuda de outras pessoas.

\section{DISCUSSÃO}

O presente estudo procurou estimar, para os idosos paulistanos, como serão vividos os anos remanescentes em relação à incapacidade funcional e à dependência. Utilizando os dados de mortalidade da população do Município de São Paulo, associados com as taxas de prevalência de incapacidade funcional e dependência, foram construídas tábuas de vida que permitiram esti-
FIGURA 4. Proporção de anos a serem vividos com incapacidade funcional e sem dependência e com incapacidade funcional e dependência em relação à expectativa de vida total por sexo e idades exatas, Município de São Paulo (SP), Brasil, 2000a

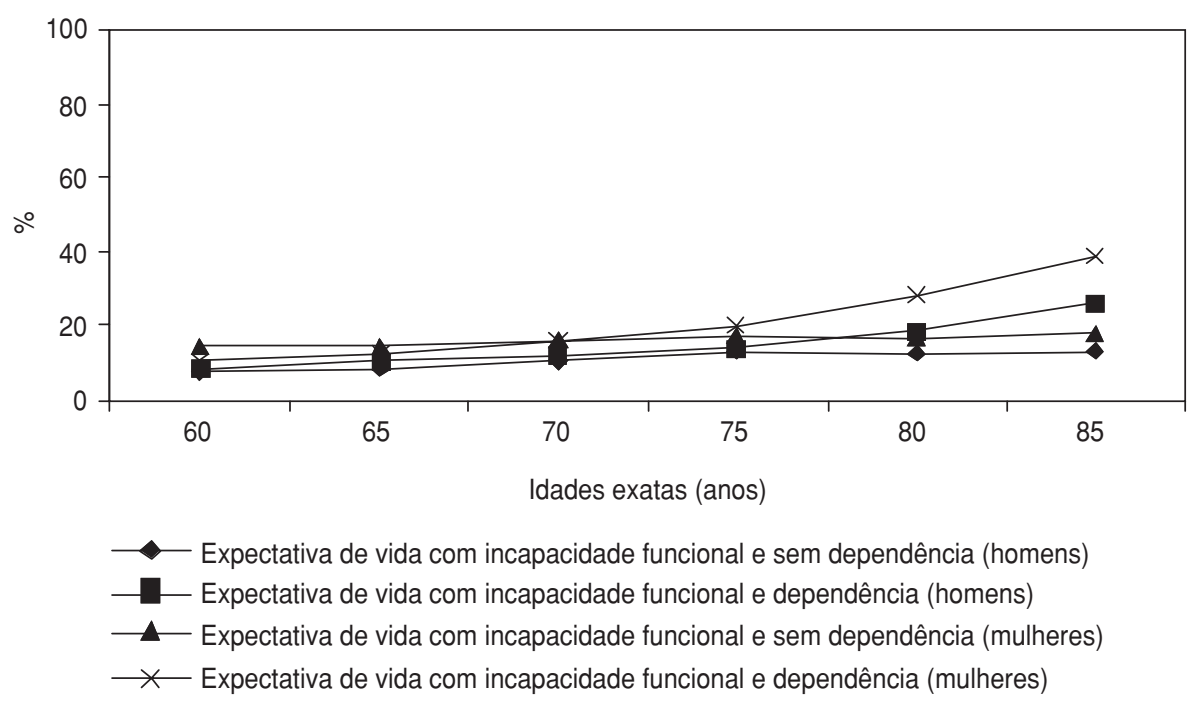

Fonte dos dados básicos para o cálculo das expectativas: Instituto Brasileiro de Geografia e Estatística (IBGE) (11, 12), Fundação Sistema Estadual de Análise de Dados (SEADE) (13) e Projeto Saúde, Bem-Estar e Envelhecimento na América Latina e Caribe (SABE) (14).

ancapacidade funcional: dificuldade de realizar uma ou mais atividades de vida diária (vestir-se, comer, tomar banho, ir ao banheiro, deitar-se e levantar da cama e atravessar um cômodo da casa). Dependência: necessidade de auxílio para realizar pelo menos uma atividade.

FIGURA 5. Proporção de anos a serem vividos com incapacidade funcional, decompostos de acordo com presença ou ausência de dependência, por sexo e idades exatas, Município de São Paulo (SP), Brasil, 2000a
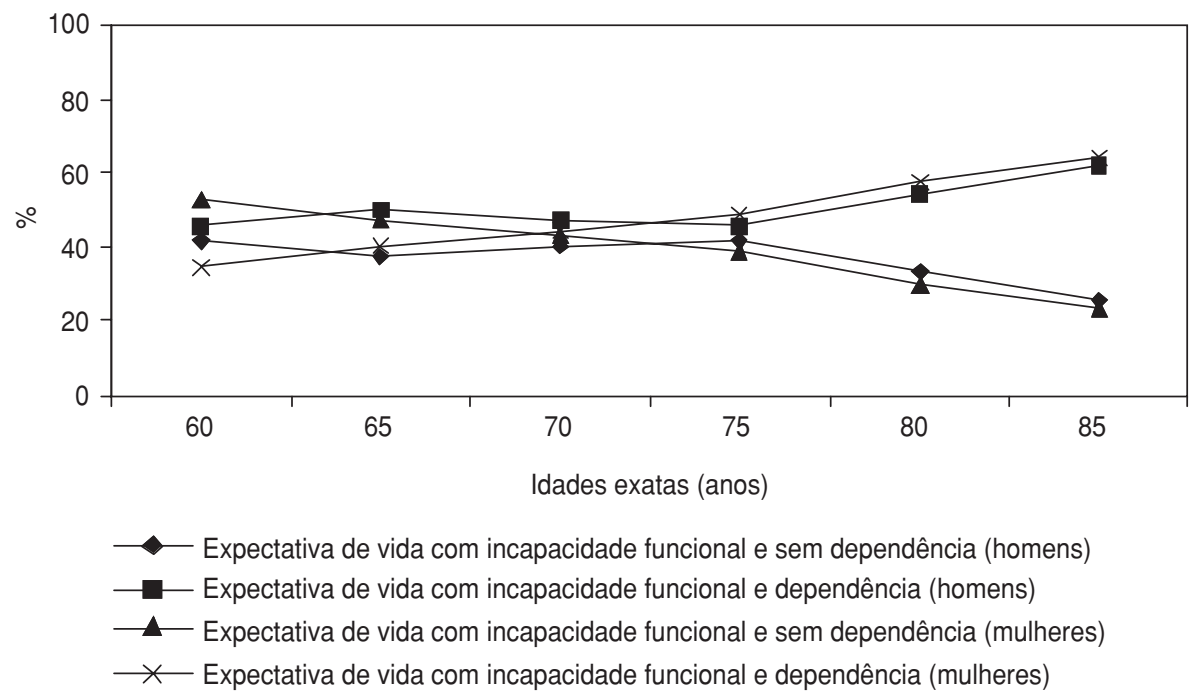

Fonte dos dados básicos para o cálculo das expectativas: Instituto Brasileiro de Geografia e Estatística (IBGE) (11, 12), Fundação Sistema Estadual de Análise de Dados (SEADE) (13) e Projeto Saúde, Bem-Estar e Envelhecimento na América Latina e Caribe (SABE) (14).

a Incapacidade funcional: dificuldade de realizar uma ou mais atividades de vida diária (vestir-se, comer, tomar banho, ir ao banheiro, deitar-se e levantar da cama e atravessar um cômodo da casa). Dependência: necessidade de auxílio para realizar pelo menos uma atividade. 
mar, para o ano de 2000, a expectativa de vida livre de incapacidade funcional, a expectativa de vida com incapacidade funcional, a expectativa de vida com incapacidade funcional e sem dependência e a expectativa de vida com incapacidade funcional e dependência.

As estimativas feitas neste trabalho são baseadas em dados de prevalência de incapacidade funcional e dependência e taxas de mortalidade da população em 2000, partindo dos pressupostos de que essas se manterão constantes ao longo do tempo e de que a mortalidade entre pessoas com e sem incapacidade funcional e dependência é semelhante, o que não necessariamente ocorre. Sabe-se que novos investimentos em cuidados e avanços tecnológicos podem permitir, por exemplo, uma queda nas taxas de prevalência (por meio do aumento das taxas de cura ou da redução da incapacidade) e nas taxas de mortalidade, possibilitando que a população não apenas viva mais, como também viva com mais qualidade, com um número maior de anos sem incapacidade funcional e dependência. Além disso, o método não permite avaliar transições de uma condição de saúde para outra, como por exemplo, o retorno de um indivíduo incapacitado funcionalmente e dependente para melhores condições de funcionalidade, o que só é analisado no modelo multiestado, com o uso de dados longitudinais de incidência. No entanto, caso não existam alterações repentinas nas taxas de prevalência e nas taxas de mortalidade, acredita-se que as estimativas aqui apresentadas sejam um reflexo da população idosa paulistana em 2000, e que possam servir de suporte no planejamento de políticas de saúde e sociais para essa população.

Uma das limitações do presente estudo é a não-inclusão de idosos institucionalizados no cálculo das taxas de prevalência de incapacidade funcional e dependência, uma vez que as entrevistas do Projeto SABE foram realizadas apenas com idosos da COmunidade. Sabe-se que, entre os institucionalizados, são comuns a dependência, as limitações e a falta de autonomia (16). Entretanto, como pro- vavelmente os institucionalizados representem apenas uma pequena proporção dos idosos, acredita-se que, mesmo se eles fossem incluídos na amostra, as taxas de prevalência não sofreriam alterações a ponto de comprometer os resultados relatados.

A mortalidade diferencial por sexo tem sido apontada como o principal fator explicativo da diferença em relação ao número de anos a serem vividos por homens e mulheres a partir de uma certa idade (17). As estimativas dos anos a serem vividos indicaram que as mulheres apresentam maior expectativa de vida do que os homens, mas passam uma maior proporção de anos com incapacidade funcional e, quando se encontram nessa condição, vivem um período proporcionalmente maior com dependência. Ainda que a vantagem feminina no que tange à esperança de vida em cada idade tenha sido consistentemente verificada em diversos estudos sobre a população idosa $(18,19)$, a maior fragilidade das mulheres mais idosas no que se refere à incapacidade funcional e dependência parece ser o resultado mais relevante deste trabalho, e vem adicionar aos estudos recentes que encontraram resultados semelhantes $(10,20,21)$.

Nesse sentido, a desvantagem feminina poderia ser atribuída à diferença de oportunidade que os homens e as mulheres tiveram ao longo da vida no que diz respeito às condições econômicas, sociais e culturais $(22,23)$. Não obstante, esse argumento deve ser considerado com reservas, uma vez que implicaria uma esperança de vida maior para os homens, a cada idade, ao longo de quase todo o ciclo de vida, o que não acontece nas populações humanas. O que parece razoável, portanto, é que as taxas de mortalidade mais elevadas dos homens em idades mais jovens poderiam permitir que, na velhice, a composição do grupo etário masculino fosse mais favorável do que a do feminino, devido a um mecanismo de seleção (24). Isso resultaria em um grupo heterogêneo, com um número maior de idosas fragilizadas e suscetíveis do que de idosos. Resultados como os do presente estudo reforçam a importância de se considerar as diferenças entre os sexos em relação à demanda por cuidado. Cabe observar, no entanto, que, em termos absolutos, a longevidade das mulheres é uma vantagem, pois pode permitir que elas tenham mais tempo para se prevenir contra possíveis incapacidades funcionais, postergando ao máximo o período de dependência e atenuando sua intensidade.

Se as políticas sociais e de saúde conseguirem promover a capacidade funcional da população idosa e construir um sistema adequado de suporte, aumentará enormemente a chance de uma velhice saudável (25). O desenvolvimento de programas na época em que a incapacidade funcional ainda não se instalou pode permitir que a pessoa envelheça de forma mais saudável, o que irá se refletir diretamente em sua condição de saúde futura. Ao mesmo tempo, na fase em que as perdas funcionais estão presentes, mas o indivíduo se mantém independente, as políticas voltadas ao tratamento desses idosos podem favorecer as melhoras na capacidade funcional e prevenir a dependência, por meio, por exemplo, da facilitação de desempenho das atividades, do replanejamento ambiental, da potencialização das atividades remanescentes e da melhora nas condições musculoesqueléticas. Já no período em que a dependência se alojou, as políticas oficiais de apoio ao idoso e à sua família podem permitir que se retarde a institucionalização, garantindo a permanência do idoso em sua casa e comunidade.

É importante ressaltar, ainda, que os problemas de saúde enfrentados pelos idosos são o resultado de um acúmulo de experiências passadas, dos cuidados com a saúde, das condições de moradia, educação, alimentação e higiene, da prática de atividade física, das oportunidades perdidas ou aproveitadas. Assim, preocupar-se com a saúde do idoso de amanhã significa investir na saúde de todas as idades. Além disso, é necessário investir naqueles que irão prestar cuidados ao idoso, sejam eles familiares ou profissionais de saúde. A família, que tradicionalmente tem oferecido apoio funcional, material e afetivo aos idosos, 
deve ser foco de atenção por parte dos governantes. Adicionalmente, em relação ao profissional de saúde, é fundamental o investimento no seu treinamento, para que sejam observadas todas as peculiaridades que o cuidado ao idoso demanda, garantindo tratamento efetivo, com menores custos e maior qualidade.

Também é essencial o desenvolvimento contínuo de pesquisas e de técnicas de tratamento direcionadas à população idosa. Como base para tais iniciativas, projetos como o SABE são importantes para revelar as demandas reais da população idosa e devem ser realizados com amostras representa- tivas de outras regiões do Brasil. Os resultados descritos aqui se aplicam apenas à população do Município de São Paulo e não podem ser extrapolados para o restante do país; contudo, podem servir de referência em termos de políticas públicas, respeitando-se as especificidades de cada região.

Ainda sobre o SABE, cabe lembrar que a base de dados permite obter as prevalências de incapacidade funcional e dependência para outros seis municípios da América Latina e do Caribe, além de São Paulo. Assim, outros estudos, seguindo a mesma metodologia descrita aqui, poderiam comparar os diferentes municípios. Adicional- mente, estudos futuros poderiam enfocar as estimativas de expectativa de vida com e sem incapacidade funcional e dependência em relação a aspectos como escolaridade, nível socioeconômico e raça. Esse tipo de informação é essencial para estimar a necessidade de serviços de apoio aos idosos dependentes e, ao mesmo tempo, produzir esforços para prevenir a dependência nos casos em que a incapacidade funcional já está instalada.

Agradecimentos. Este trabalho foi realizado com o apoio da Fundação de Amparo à Pesquisa do Estado de Minas Gerais.

\section{REFERÊNCIAS}

1. Portrait F, Maarten L, Degg D. Life expectancies in specific health states: results from a joint model of health status and mortality of older persons. Demography. 2001;38(4): 525-36.

2. Sanders BS. Measuring community health levels. Am J Public Health. 1964;54(7): 1063-70.

3. Sullivan DF. A single index of mortality and morbidity. HSMHA Health Rep. 1971;86(4): 347-54.

4. Bone MR. International efforts to measure health expectancy. J Epidemiol Community Health. 1992;46(6):555-8.

5. Robine JM, Romieu I, Cambois E. Health expectancy indicators. Bull World Health Organ. 1999;77(2):181-5.

6. Manton KG, Land KC. Active life expectancy estimates for U.S. elderly population: a multidimensional continuous-mixture model of functional change applied to completed cohorts. Demography. 2000;37(3):253-65.

7. Jagger $C$. Health expectancy calculation by the Sullivan method: a practical guide. Madison: Nihon University Population Research Institute; 1999. (NUPRI Research Paper 68).

8. Mathers CD, Robine JM. How good is Sullivan's method for monitoring changes in population health expectancies? J Epidemiol Community Health. 1997;51(1):80-6.

9. Bone MR, Bebbington AC, Nicolaas G. Policy applications of health expectancy. J Aging Health. 1998;10(2):136-53.

10. Agree EM. The influence of personal care and assistive devices on the measurement of disability. Soc Sci Med. 1999;48(4):427-43.

11. Instituto Brasileiro de Geografia e Estatística. Censo demográfico: 1991. Rio de Janeiro: IBGE; 1991.
12. Instituto Brasileiro de Geografia e Estatística. Censo demográfico: 2000. Rio de Janeiro: IBGE; 2001.

13. Fundação Sistema Estadual de Análise de Dados. Óbitos ocorridos no município de São Paulo, 1999, 2000 e 2001. São Paulo: Fundação SEADE.

14. Peláez M, Palloni A, Albala C, Alfonso JC, Ham-Chande R, Hennis A, et al. Survey on aging, health and well-being, 2000. Washington, D.C.: Pan American Health Organization, World Health Organization; 2003.

15. Lebrão ML, Duarte YAO. O projeto SABE no Brasil: uma abordagem inicial. Brasília: Organização Pan-Americana da Saúde; 2003.

16. Cerqueira MBR. Envelhecimento populacional e população institucionalizada: um estudo de caso dos asilos do município de Montes Claros [dissertação de mestrado]. Belo Horizonte: Universidade Federal de Minas Gerais; 2003.

17. Berquo E. Algumas considerações demográficas sobre o envelhecimento da população no Brasil. Em: Anais do I Seminário internacional sobre envelhecimento populacional: uma agenda para o fim do século. Brasília: Ministério da Previdência Social, Secretaria da Assistência Social; 1996. Pp. 16-34.

18. Guralnik JM, Balfour JL, Volpato S. The ratio of older women to men: historical perspectives and cross-national comparisons. Aging (Milano). 2000;12(2):65-76.

19. Trovato F, Lalu NM. Narrowing sex differentials in life expectancy in the industrialized world: early 1970 's to early 1990 's. Soc Biol. 1996;43(1-2):20-37.

20. Instituto Brasileiro de Geografia e Estatística. Censo demográfico 2000: características gerais da população, resultados da amostra. Rio de Janeiro: IBGE; 2003.

21. Baptista DBDA. Idosos no município de São Paulo: expectativa de vida ativa e perfis multidimensionais de incapacidade a partir da SABE [dissertação de mestrado]. Belo Horizonte: Universidade Federal de Minas Gerais; 2003.

22. Goldani AM. Mulheres e envelhecimento: desafios para novos contratos intergeracionais e de gênero. Em: Camarano AA, org. Muito além dos 60: os novos idosos brasileiros. Rio de Janeiro: Instituto de Pesquisa Econômica Aplicada; 1999. Pp. 75-113.

23. Barreto SM, Giatti L, Uchoa E, Lima-Costa MF. Gênero e desigualdades em saúde entre idosos brasileiros. Em: Anais da Oficina de trabalho sobre desigualdades sociais e de gênero em saúde de idosos no Brasil. Ouro Preto: Núcleo de Estudos em Saúde Pública e Envelhecimento, Centro de Pesquisas René Rachou da Fundação Oswaldo Cruz, Faculdade de Medicina da Universidade Federal de Minas Gerais; 2002. Pp. 59-69.

24. Perls T, Kunkel LM, Puca AA. The genetics of exceptional human longevity. J Mol Neurosci. 2002;19(1-2):233-8.

25. Paschoal SMP. Autonomia e independência. Em: Papaleo-Netto M, org. Gerontologia: a velhice e o envelhecimento em visão globalizada. São Paulo: Atheneu; 2002. Pp. 311-23.

Manuscrito recebido em 29 de junho de 2004. Aceito em versão revisada em 28 de setembro de 2004. 
ABSTRACT Objective. For persons 60 years of age or older living in the city of São Paulo, Brazil, in the year 2000 to estimate four characteristics: (1) life expectancy free of functional disability, (2) life expectancy with functional disability, (3) life expectancy with func-

Life expectancy with functional disability in elderly persons in São Paulo, Brazil tional disability but without dependence, and (4) life expectancy with functional disability and dependence.

Methods. The estimates of the four characteristics were calculated by means of a life table constructed based on the method proposed by Sullivan. The basic data used for the calculations were the elderly population estimated for the city of São Paulo as of mid-2000, obtained from the demographic censuses of 1991 and 2000, and deaths in the elderly population, obtained from the State Data Analysis System Foundation (Fundação Sistema Estadual de Análise de Dados, or SEADE) of the state of São Paulo. The prevalences of functional disability and of functional dependence were calculated based on data concerning activities of daily living collected in the city of São Paulo as part of a project called Health, Well-being, and Aging in Latin America and the Caribbean (the "SABE project"). The activities of daily living considered were: dressing, eating, bathing, using the bathroom, lying down on the bed and getting up from it, and walking across a room. Functional disability was defined as difficulty in performing one or more of the activities of daily living. Dependence was defined as the need for help in performing at least one of the activities of daily living.

Results. In 2000, 60-year-old men from the city of São Paulo could expect to live, on average, 17.6 years, of which 14.6 years $(83 \%)$ would be free of functional disability. Women of the same age could expect to live 22.2 years, of which 16.4 years $(74 \%)$ would be free of functional disability. Men would have a functional disability and be dependent on others for 1.6 years (9\%), while the comparable period for women would be 2.5 years $(11 \%)$.

Conclusions. Despite their longer life expectancy, the women faced more years with functional disability. The number of years with functional disability and dependence was also higher for the women. Public policies should take into account the differing needs of elderly women and of elderly men as well as other specific characteristics of this older population.

Keywords Population dynamics, quality of life, health services for the aged. 